\title{
Estabelecimento de zonas de melhoramento para clones de eucalipto no Rio Grande do Sul
}

\author{
Establishment of improvement areas for \\ eucalyptus clones in Rio Grande do Sul, Brazil
}

\author{
Andrei Caíque Pires Nunes', Gleison Augusto Santos², Marcos Deon Vilela Resende ${ }^{3}$, \\ Luciana Duque Silva ${ }^{4}$, Antonio Higa ${ }^{5}$ e Teotônio Francisco Assis ${ }^{6}$
}

\begin{abstract}
Resumo
A presente pesquisa objetivou estudar a seleção indireta e o estabelecimento de zonas de melhoramento utilizando-se o caráter densidade básica-produtividade de clones de eucalipto em quatro ambientes do estado do Rio Grande do Sul (Cambará, Fortaleza, Capivara e São João). Os ensaios foram conduzidos nas áreas da empresa CMPC Celulose Riograndense. No ano de 2007, foi implantada uma rede de testes clonais com 864 clones de eucalipto. Aos três anos de idade, foram mensurados o diâmetro à altura do peito (DAP), a altura total das árvores, o volume e a profundidade de penetração do Pilodyn (Db), para estimação indireta da densidade básica da madeira. Um índice de peso de madeira (DAPxDb) foi criado. Herdabilidades individuais de 0,0770 para altura a 0,5928 para $\mathrm{Db}$ foram estimadas. A correlação genética entre os caracteres DAP e volume foi elevada $(0,97)$. Com isso, a eficiência na seleção indireta foi calculada $(1,05)$ e constatou-se que é vantajoso obter ganhos indiretos em volume pela seleção em DAP. A correlação genética entre os ambientes variou de 0,4014 a 0,9315 para os caracteres avaliados. $O$ índice $\mathrm{DAPxDb}$ apresentou interação genótipos $\mathrm{x}$ ambientes significativa, com correlação genética entre os ambientes de 0,6743. Assim, procedeu-se o estabelecimento das zonas de melhoramento para este caráter. A partir da análise de interação dos ambientes par a par, duas zonas de melhoramento foram definidas para DAPxDb (Cambará-Fortaleza-São João e Capivara). A medição precisa do DAP juntamente com a densidade básica no índice $\mathrm{DAPxDb}$ foi fundamental na obtenção de apenas duas zonas de melhoramento, sem a necessidade do uso da característica volume. Dessa forma, houve otimização da seleção dos genótipos, dado que a medição do DAP é mais rápida e precisa em relação à estimação do volume, que envolve, além de DAP, o caráter altura.
\end{abstract}

Palavras-chave: Estratificação ambiental; Pilodyn; Índice DAPxDb.

\begin{abstract}
The present study aimed to evaluate the indirect selection and establishment of improvement areas, using the basic wood density-productivity character of Eucalyptus clones in four environments located in the state of Rio Grande do Sul, Brazil (Cambará, Fortaleza, Capivara and São João). The test was conducted in the areas of CMPC Celulose Riograndense. In 2007, a network of clonal tests with 864 clones of Eucalyptus was deployed. At three years of age, the characters diameter at breast height (DBH), total height and volume were measured. The depth of Pilodyn penetration was used, for indirect measurement of wood basic density $(\mathrm{Bd})$. A DBHxBd index of wood weight was created. The results showed individual heritability ranging from 0.0770 to total height and 0.5928 for $\mathrm{Bd}$. The genetic correlation between $\mathrm{DBH}$ characters and volume was high (0.97). Thus, the efficiency of indirect selection was calculated (1.05). Therefore, it is advantageous to obtain indirect gains in volume through selection by $\mathrm{DBH}$. The genetic correlation between environments ranged from 0.4014 to 0.9315 for the traits. The DBHxBd index showed significant genotype $\mathrm{x}$ environment interaction with genetic correlation of 0.6743 between the four environments. So,

\footnotetext{
${ }^{1}$ Doutorando em Genética e Melhoramento. UFV - Universidade Federal de Viçosa. Avenida Peter Henry Rolfs, s/n - Campus Universitário - Centro - 36570000 - Viçosa, MG, Brasil. E-mail: andreicaique@yahoo.com.br. Rolfs, s/n - Campus Universitário - Centro - 36570000 - Viçosa, MG, Brasil. E-mail: gleisons.ufv@gmail.com. domínio Vila Castela - 34000-000 - Nova Lima, MG, Brasil. E-mail: marcos.deon@gmail.com. Luiz de Queiroz. Av. Pádua Dias - 13418-900 - Piracicaba, SP, Brasil. E-mail: lucianaduques@yahoo.com.br.

${ }^{5}$ Professor Adjunto do Departamento de Ciências Florestais. UFPR - Universidade Federal do Paraná, Setor de Ciências Agrárias. Rua Lothário Meissner 900 - Jardim Botânico - 80210170 - Curitiba, PR, Brasil. E-mail: antonio.higa@gmail.com. ${ }^{6}$ Consultor Interno. Klabin Companhia Rio Grande de Celulose do Sul. Rua São Geraldo, 1680 - Caixa-postal: 108 92500-000 - Guaiba, RS, Brasil. E-mail: assisteo@terra.com.br.
}

${ }^{2}$ Professor Doutor do Departamento de Engenharia Florestal. UFV - Universidade Federal de Viçosa. Avenida Peter Henry

${ }^{3}$ Consultor em Melhoramento Florestal. Assistech Tecnologia em Melhoramento. Avenida Reis Magos número, 587 - Con-

${ }^{4}$ Professora Doutora do Departamento de Ciências Florestais. USP - Universidade de São Paulo / ESALQ - Escola Superior
\end{abstract}


the establishment of improvement areas was performed for this character. From the interaction analysis of the environments in pairs, two improvement areas were defined for the character (Cambará-Fortaleza-São João and Capivara). Accurate measurement of $\mathrm{DBH}$ with the basic wood density in $\mathrm{DBH}$ Bd index was crucial to obtain only two improvement areas without the use of the characteristic volume. Thus, there was genotype selection optimization, since the measurement of DHB is faster and more accurate in relation to volume, which involves, in addition to $\mathrm{DBH}$, the total height character.

Keywords: Environmental stratification; Pilodyn; DBHxBd index.

\section{INTRODUÇÃO}

Estudos de interação genótipos x ambientes ( $\mathrm{x}$ x A) são fundamentais na obtenção de ganhos genéticos, constituindo uma das etapas mais importantes num programa de melhoramento florestal (HARDNER et al., 2011). Contudo, a simples identificação de interação G x A "per se" não proporciona informações completas sobre o comportamento de cada genótipo frente às variações ambientais, sendo necessário o conhecimento da magnitude dessa interação. Para isso, análises de adaptabilidade e estabilidade genotípica devem ser realizadas, tornando possível a identificação de genótipos com comportamento constante e/ou que sejam responsivos às variações ambientais, em condições específicas ou amplas de acordo com o interesse do melhorista (CRUZ; REGAZZI, 1994; HARDNER et al., 2011). Apesar da relevância desses estudos, trabalhos que abordam a análise da interação G x A considerando características de qualidade da madeira são extremamente escassos no Brasil.

A qualidade da madeira pode ser definida como o conjunto de características físicas, químicas e anatômicas que uma árvore/parte dela possui, e que lhe permite atender os requisitos necessários para ser transformada em distintos produtos finais (MITCHELL, 1961). Na indústria do papel e celulose, a qualidade do lenho é caracterizada com base em análises tecnológicas, dentre elas, cita-se: determinação da densidade básica, tamanho das fibras, teor de lignina, teor de extrativos, rendimento da polpação e consumo de madeira para produção de celulose (GOMIDE et al., 2010). Assim, a realização dessas análises em programas de melhoramento do eucalipto torna-se indispensável para a determinação da qualidade da madeira dos materiais genéticos testados, objetivando a maximização de ganhos na produção de celulose (CAIXETA et al., 2003; GOMIDE et al., 2010; PROTÁSIO et al., 2014).

Análises de adaptabilidade, estabilidade e estudos de qualidade da madeira assumem relevante importância em um programa de melhoramento florestal, assim como a estratificação ambiental. Este tipo de análise caracteriza-se como uma ferramenta fundamental na recomendação de materiais genéticos nos casos em que a interação G x A é significativa (VENCOVSKY; BARRIGA, 1992; CRUZ et al., 2004). Dessa forma, com o intuito de amenizar os efeitos da interação do tipo complexa, pode-se subdividir a área total do experimento em zonas de melhoramento. O termo zonas de melhoramento foi reportado por Resende (2005) para caracterizar as áreas nas quais a interação G x A não é do tipo complexa, ou seja, cada zona demandará um programa de melhoramento específico e os ganhos genéticos dentro dessas zonas serão maximizados. Com isso, o estabelecimento dessas unidades de produção, aliado às análises de adaptabilidade e estabilidade, são fatores cruciais para o sucesso de um programa de melhoramento florestal, dado que permitem um estudo detalhado do comportamento dos genótipos nos diferentes ambientes testados.

Assim como os estudos de estratificação ambiental, a resposta correlacionada entre caracteres é um fator importante, que pode ser utilizado para otimização da seleção. Na presença de correlação genética de alta magnitude entre dois caracteres, por exemplo, a alteração em um caráter, via seleção, promove alterações significativas no outro caráter correlacionado (RESENDE, 2002). A resposta indireta assume grande importância, principalmente, nos casos em que a medição de determinado caráter é difícil ou está sujeita a erros de medição e quando a herdabilidade desse caráter é baixa (GOLDENBERG, 1968; FALCONER, 1987). Dessa forma, a seleção indireta de caracteres em eucalipto, a exemplo da obtenção de ganhos para volume por meio da medição do diâmetro à altura do peito, constitui uma forma eficiente de praticar seleção de genótipos superiores.

Considerando esses aspectos, o presente estudo objetivou analisar a resposta indireta de caracteres em eucalipto por meio de correlações genéticas e estabelecer zonas de melhoramento no estado do Rio Grande do Sul, pelo uso dos caracteres de produtividade em associação com uma 
característica de qualidade da madeira, a densidade básica. Ademais, objetivou-se, também, estudar a adaptabilidade e a estabilidade dos clones de eucalipto.

\section{MATERIAL E MÉTODOS}

\section{Áreas experimentais}

Os experimentos foram conduzidos nas áreas da empresa CMPC Celulose Riograndense, nos municípios de Minas do Leão (Horto Florestal Cambará), Encruzilhada do Sul (Horto Florestal Capivara), Dom Feliciano (Horto Florestal Fortaleza) e Vila Nova do Sul (Horto Florestal São João), todos localizados no estado do Rio Grande do Sul. As árvores foram plantadas no espaçamento de $3,5 \mathrm{~m}$ x 2,6 m, os dados edafoclimáticos e as coordenadas geográficas de cada local estudado podem ser encontrados na Tabela 1 .

Tabela 1. Localização geográfica e condições edafoclimáticas dos quatro ambientes do estado do Rio Grande do Sul utilizados para experimentação.

Table 1. Geographical location and edaphic-climatic conditions of four environments of the State of Rio Grande do Sul, Brazil, used for the trials.

\begin{tabular}{|c|c|c|c|c|}
\hline & Cambará & Capivara & Fortaleza & São João \\
\hline Coordenadas Geográficas & $\begin{array}{l}\text { Latitude: } 30^{\circ} 11^{\prime} 09^{\prime \prime} \mathrm{S} ; \\
\text { Longitude } 52^{\circ} 00^{\prime} 10^{\prime \prime} \mathrm{W} ;\end{array}$ & $\begin{array}{l}\text { Latitude: } 30^{\circ} 27^{\prime} 19^{\prime \prime} \mathrm{S} ; \\
\text { Longitude: } 52^{\circ} 39^{\prime} 53^{\prime \prime} \mathrm{W} ;\end{array}$ & $\begin{array}{l}\text { Latitude: } 30^{\circ} 29^{\prime} 45^{\prime \prime} \text { S; } \\
\text { Longitude: } 52^{\circ} 19^{\prime} 35^{\prime \prime} \text { W; }\end{array}$ & $\begin{array}{l}\text { Latitude: } 30^{\circ} 14^{\prime} 46^{\prime \prime} \mathrm{S} \text {; } \\
\text { Longitude: } 53^{\circ} 49^{\prime} 7^{\prime \prime} \mathrm{W} ;\end{array}$ \\
\hline Altitude (metros) & 141 & 250 & 378 & 301 \\
\hline Município & Minas do Leão & Encruzilhada do Sul & Dom Feliciano & Vila Nova do Sul \\
\hline Ocupação anterior & $\begin{array}{l}\text { Plantio de Eucalyptus } \\
\text { (Área de reforma) }\end{array}$ & Pastagem & Pastagem & Pastagem \\
\hline Tipo de Solo & $\begin{array}{l}\text { Argissolo Vermelho } \\
\text { distrófico nitossólico - } \\
\text { textura argilosa }\end{array}$ & $\begin{array}{l}\text { Argissolo Vermelho } \\
\text { distrófico abrúptico - tex- } \\
\text { tura arenosa/argilosa }\end{array}$ & $\begin{array}{c}\text { Argissolo Vermelho } \\
\text { Amarelo distrófico típico } \\
\text { - textura média/argilosa }\end{array}$ & $\begin{array}{c}\text { Argissolo Vermelho } \\
\text { distrófico típico - textura } \\
\text { média/argilosa }\end{array}$ \\
\hline Fertilidade & média a alta & média a alta & média a alta & média a baixa \\
\hline Profunidade do solo $(\mathrm{mm})$ & profundo & médio & raso & profundo \\
\hline Relevo & plano suave ondulado & ondulado & ondulado & plano suave ondulado \\
\hline $\begin{array}{l}\text { Mudança textural } \\
\text { (perfil do solo) }\end{array}$ & leve & moderada & forte & leve \\
\hline Temperatura Média $\left({ }^{\circ} \mathrm{C}\right)$ & 17,5 & 17,0 & 16,0 & 16,8 \\
\hline $\begin{array}{l}\text { Temperatura mínima } \\
\text { absoluta }\left({ }^{\circ} \mathrm{C}\right)\end{array}$ & $-0,9$ & $-0,6$ & $-1,7$ & 0,0 \\
\hline $\begin{array}{l}\text { Temperatura máxima } \\
\text { absoluta }\left({ }^{\circ} \mathrm{C}\right)\end{array}$ & 32,3 & 33,3 & 30,7 & 34,7 \\
\hline Umidade relativa (\%) & 77,6 & 74,8 & 80,6 & 75,6 \\
\hline Pluviosidade (mm) & 1.422 & 1.368 & 1.564 & 1.133 \\
\hline $\begin{array}{l}\text { Velocidade do vento } \\
\text { (metros/segundo) }\end{array}$ & 5,5 & 5,4 & 4,9 & 6,2 \\
\hline
\end{tabular}

Fonte: Adaptado de Santos et al. (2013).

\section{Coleta de dados}

Aos três anos de idade foram mensurados o diâmetro à altura do peito (DAP), em centímetros, e a altura total das árvores, em metros. O DAP foi mensurado com o auxílio de uma fita diamétrica e a altura foi obtida com o uso do relascópio.

Para o cálculo do volume sem casca foi utilizado o modelo de Leite et al. (1995), conforme apresentado abaixo:

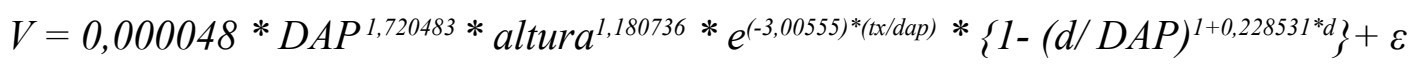

Em que: DAP: diâmetro a 1,3 metro de altura; altura: altura total; $\mathrm{tx}=0$, para volume com casca e 1 para volume sem casca; d: diâmetro comercial superior; = erro experimental.

Para a estimação indireta da densidade básica, o método do Pilodyn foi utilizado. Esse método constitui na penetração de uma agulha de aço com 2,5 mm de diâmetro na face exterior do tronco, impulsionada por uma mola com força constante de 6 joules (GREAVES et al., 1996). Para tal, foi necessário descascar previamente uma secção retangular do tronco de aproximadamente $3 \mathrm{~cm}$ de comprimento, por $2 \mathrm{~cm}$ de largura na altura de 1,3 m do solo (DAP), de forma que a agulha de aço pe- 
netrasse apenas no lenho da árvore. Depois de encostado o aparelho ao tronco, disparou-se o gatilho e mediu-se a penetração da agulha no lenho da árvore em milímetros, através de um visor numérico presente no aparelho. O Pilodyn foi utilizado, duas vezes, nas faces norte e sul de cada árvore. Caso o resultado de algum dos dois acionamentos fosse diferente, era realizado um terceiro acionamento e descartava-se o número discrepante. Para efeito das análises, o número considerado foi a média dos dois acionamentos realizados. Assim, a densidade básica estimada indiretamente está representada por $\mathrm{Db}$ (1/profundidade de penetração do pilodyn), que é o inverso de profundidade de penetração, dado que quanto maior essa medida menor é a densidade do lenho (GREAVES et al., 1996).

\section{Índice DAPxDb}

Um índice de peso de madeira DAPxDb foi criado. Dessa forma, ao unir a densidade básica da madeira a um critério de crescimento, objetivou-se tornar as análises mais acuradas e maximizar o ganho genético:

$$
D A P \times D b=\left(\frac{D A P}{S_{D A P}}\right) \times\left(\frac{D b}{S_{D b}}\right)
$$

Em que: $\mathrm{DAPxDb}$ = índice de peso de madeira; $\mathrm{Db}=$ densidade básica estimada indiretamente pelo Pilodyn; $\mathrm{DAP}=$ diâmetro à altura do peito; $\mathrm{S}_{\mathrm{DAP}}=$ desvio padrão da variável diâmetro à altura do peito; $\mathrm{S}_{\mathrm{Db}}=$ desvio padrão da variável densidade básica.

Pesos econômicos relativos iguais foram atribuídos aos dois caracteres (DAP e Db).

\section{Delineamentos estatísticos e análise de estabilidade e adaptabilidade}

Uma rede de testes clonais com 864 clones de eucalipto foi implantada no ano de 2007, em quatro ambientes do estado do Rio Grande do Sul. Como testemunha comparativa, foi utilizado o principal clone comercial atualmente plantado pela empresa (clone 32864). Em cada sítio um experimento no delineamento de blocos ao acaso foi estabelecido, com parcela de árvore única e 30 repetições. O modelo estatístico para análise dessa rede experimental em vários ambientes (RESENDE, 2002), considerando a tomada de uma observação por parcela, é dado por:

$$
y=X r+Z g+H b+W g e+e
$$

Em que:

y, r, g, b, ge, e = vetores de dados, de efeitos de repetições (fixos), de efeitos genotípicos (aleatório), dos efeitos de bloco (aleatório), de efeitos da interação G x A (aleatório) e de erros aleatórios, respectivamente.

$\mathrm{X}, \mathrm{Z}, \mathrm{H}$ e $\mathrm{W}=$ matrizes de incidência para $\mathrm{r}, \mathrm{g}$, b e ge, respectivamente.

Distribuições e estruturas de médias e variâncias e equações de modelo misto:

$$
\left[\begin{array}{cccc}
X^{\prime} X & X^{\prime} Z & X^{\prime} H & X^{\prime} W \\
Z X & Z^{\prime} Z+I \lambda_{4} & Z^{\prime} H & Z^{\prime} W \\
H X & H Z & H H+I \lambda_{2} & H W \\
W X & W Z & W H & W W+I \lambda_{3}
\end{array}\right]\left[\begin{array}{c}
\hat{r} \\
\hat{b} \\
\hat{g} \\
\hat{g} e
\end{array}\right]=\left[\begin{array}{l}
X^{\prime} y \\
Z^{\prime} y \\
H y \\
W y
\end{array}\right] E\left[\begin{array}{c}
y \\
g \\
b \\
g e \\
g \\
e
\end{array}\right]=\left[\begin{array}{c}
X r \\
0 \\
0 \\
0 \\
0
\end{array}\right]=V a r\left[\begin{array}{c}
g \\
b \\
g e \\
e
\end{array}\right]=\left[\begin{array}{cccc}
I \sigma_{g}^{2} & 0 & 0 & 0 \\
0 & I \sigma_{b}^{2} & 0 & 0 \\
0 & 0 & I \sigma_{g^{2}}^{2} & 0 \\
0 & 0 & 0 & I \sigma_{z}^{2}
\end{array}\right]
$$

Em que:

$$
\lambda_{1}=\frac{\sigma_{\varepsilon}^{2}}{\sigma_{g}^{2}}=\frac{1-h_{g}^{2}-c_{b}^{2}-c_{g \varepsilon}^{2}}{h_{g}^{2}} ; \quad \lambda_{2}=\frac{\sigma_{\varepsilon}^{2}}{\sigma_{b}^{2}}=\frac{1-h_{g}^{2}-c_{b}^{2}-c_{g \varepsilon}^{2}}{c_{b}^{2}} . \quad \lambda_{3}=\frac{\sigma_{\varepsilon}^{2}}{\sigma_{g \varepsilon}^{2}}=\frac{1-h_{g}^{2}-c_{b}^{2}-c_{g \varepsilon}^{2}}{c_{g \varepsilon}^{2}}
$$

$h_{g}^{2}=\frac{\sigma_{g}^{2}}{\sigma_{g}^{2}+\sigma_{b}^{2}+\sigma_{g \varepsilon}^{2}+\sigma_{\varepsilon}^{2}}$ : herdabilidade individual no sentido amplo de parcelas individuais na repetição;

$c_{b}^{2}=\frac{\sigma_{b}^{2}}{\sigma_{g}^{2}+\sigma_{b}^{2}+\sigma_{g e}^{2}+\sigma_{\varepsilon}^{2}}$ : correlação devida ao ambiente comum do bloco;

$c_{g e}^{2}=\frac{\sigma_{g e}^{2}}{\sigma_{g}^{2}+\sigma_{b}^{2}+\sigma_{g e}^{2}+\sigma_{\varepsilon}^{2}}$ : coeficiente de determinação dos efeitos da interação G x A; 
$\sigma_{g}^{2}=$ variância genotípica; $\sigma_{b}^{2}=$ variância ambiental entre blocos; $\sigma_{g \varepsilon}^{2}=$ variância da interação $\mathrm{G}$ $\mathrm{x} \mathrm{A} ; \sigma_{\varepsilon}^{2}=$ variância residual entre parcelas;

$r_{g l o c}=\frac{\sigma_{g e}^{2}}{\sigma_{g}^{2}+\sigma_{g e}^{2}}=\frac{h_{g}^{2}}{h_{g}^{2}+c_{g e}^{2}}$ : correlação genotípica dos materiais genéticos através dos ambientes.

Estimadores de componentes de variância por REML via algoritmo EM:

$$
\begin{aligned}
\hat{\sigma}_{\varepsilon}^{2}=\left[y^{\prime} y-\hat{r}^{\prime} X^{\prime} y-\hat{g}^{\prime} Z^{\prime} y-\hat{b}^{\prime} H^{\prime} y-g \hat{e}^{\prime} W^{\prime} y\right] /[N-r(x)] \\
\left.\hat{\sigma}_{g}^{2}=\left[\hat{g}^{\prime} \hat{g}+\hat{\sigma}_{\varepsilon}^{2}(t r) C^{22}\right)\right] / q \\
\left.\hat{\sigma}_{b}^{2}=\left[\hat{b}^{\prime} b+\hat{\sigma}_{\varepsilon}^{2}(t r) C^{33}\right)\right] / s \\
\hat{\sigma}_{g^{\varepsilon}}^{2}=\left[g \hat{e}^{\prime} g \hat{e}+\hat{\sigma}_{\varepsilon}^{2}(\operatorname{tr}) C^{44}\right] / n
\end{aligned}
$$

$C^{22}, C^{33} \mathrm{e}^{C^{44}}$ advém de:

$$
C^{-1}-\left[\begin{array}{llll}
C_{11} & C_{12} & C_{13} & C_{14} \\
C_{21} & C_{22} & C_{23} & C_{24} \\
C_{31} & C_{32} & C_{33} & C_{34} \\
C_{41} & C_{42} & C_{43} & C_{44}
\end{array}\right]^{-1}=\left[\begin{array}{llll}
C^{11} & C^{12} & C^{13} & C^{14} \\
C^{21} & C^{22} & C^{23} & C^{24} \\
C^{31} & C^{32} & C^{33} & C^{34} \\
C^{41} & C^{42} & C^{43} & C^{44}
\end{array}\right]
$$

Em que:

C: matriz dos coeficientes das equações de modelo misto; tr: operador traço matricial; r(x): posto da matriz $\mathrm{X} ; \mathrm{N}, \mathrm{q}, \mathrm{s}, \mathrm{n}=$ número total de dados, de clones, de blocos e de combinações $\mathrm{G}$ x A, respectivamente.

Nesse modelo, os valores genotípicos preditos livres da interação, considerando todos os locais são dados por $\mathrm{u}+\mathrm{g}$, em que u é a média de todos os locais. Para cada local j, os valores genotípicos são preditos por $u_{j}+g+$ ge, em que $u_{j}$ é a média do local $j$. A seleção conjunta por DAPxDb estabilidade e adaptabilidade dos materiais genéticos foi baseada na estatística denominada média harmônica da performance relativa dos valores genéticos (MHPRVG) preditos, conforme descrito por Resende (2004). Com os valores genéticos preditos, foi obtida a correlação conjunta entre os ambientes e a correlação entre os locais tomados par a par. Além disso, obteve-se a correlação genética entre as variáveis analisadas. Todas as análises foram realizadas utilizando o programa de Seleção Genética Computadorizada - Selegen-REML/BLUP.

\section{Eficiência na seleção indireta em relação à direta}

Considerando o ganho genético indireto numa característica y, em função da seleção na característica $\mathrm{x}$, a eficiência na seleção indireta em relação à direta é dada pela expressão abaixo, reportada por Resende (2002):

Eficiência da seleção indireta $=\frac{\varphi_{x, y}}{h_{y}^{2}}$;

Por sua vez: $\varphi_{x, y}=r_{g x, y} \times h_{x} \times h_{y}$

Em que: $\varphi_{x, y}=$ equivale ao produto das acurácias (associadas à seleção massal, $h_{x}$ e $h_{y}$ ) e da correlação genética entre os dois caracteres $\left(r_{g x, y}\right) \cdot h^{2}=$ herdabilidade da característica y.

\section{RESULTADOS E DISCUSSÃO}

\section{Parâmetros genéticos}

Observa-se um alto valor estimado de herdabilidade no sentido amplo para o caráter $\mathrm{Db}\left(\mathrm{h}^{2} \mathrm{~g}=\right.$ 0,5928), Tabela 2. Resende (2002) classifica as magnitudes de herdabilidade individuais em: baixa, 
quando varia de 0,01 a 0,15; média ou moderada, que varia de 0,15 a 0,50; alta, quando acima de 0,50. Com isso, constata-se o elevado controle genético dessa característica e possibilidade de obtenção de ganhos genéticos significativos com a seleção de indivíduos pela sua densidade básica medida indiretamente. Não obstante ao elevado controle genético do caráter $\mathrm{Db}$, os demais valores de herdabilidade $\left(\mathrm{h}^{2} \mathrm{~g}\right)$ podem ser considerados moderados, variando de 0,1550 para volume a 0,2325 para o índice de peso de madeira DAPxDb e baixo de 0,0770 para altura (Tabela 2).

Tabela 2. Estimativas de parâmetros genéticos (REML individual), para os caracteres Db (mm), DAP (cm), altura total $(\mathrm{m})$, volume $\left(\mathrm{m}^{3} \cdot \mathrm{ha}^{-1}\right.$.ano-1) e índice DAPxDb para clones de eucalipto aos três anos de idade, avaliados na análise conjunta entre os ambientes.

Table 2. Estimates of genetic parameters (individual REML) for the characters $B d(\mathrm{~mm}), \mathrm{DBH}(\mathrm{cm})$, total height $(\mathrm{m})$, volume $\left(\mathrm{m}^{3} \cdot \mathrm{ha}^{-1}\right.$.year $\left.{ }^{-1}\right)$ and DBHxBd index, for Eucalyptus clones at three years of age, evaluated in the joint analysis between environments.

\begin{tabular}{|c|c|c|c|c|c|}
\hline & Db & DAP & Altura & Volume & DAPxDb \\
\hline$h^{2} g^{*}$ & 0,5928 & 0,1831 & 0,0770 & 0,1550 & 0,2325 \\
\hline $\mathrm{h}^{2} \mathrm{mg}^{* *}$ & 0,9529 & 0,7058 & 0,5410 & 0,6543 & 0,7812 \\
\hline Acgen** & 0,9761 & 0,8401 & 0,7355 & 0,8089 & 0,8838 \\
\hline$c^{2}$ bloc & 0,2428 & 0,0361 & 0,0868 & 0,0511 & 0,1585 \\
\hline$c^{2}$ int & 0,0329 & 0,1343 & 0,1048 & 0,1452 & 0,0945 \\
\hline rgloc & 0,9315 & 0,5677 & 0,4014 & 0,5030 & 0,6743 \\
\hline Média geral & 496,4035 & 13,27 & 14,73 & 32,4883 & 6,0398 \\
\hline CVgi\% & 12,3916 & 8,1675 & 5,0940 & 17,8915 & 12,0416 \\
\hline CVe\% & 9,7043 & 15,7106 & 16,5016 & 37,8052 & 20,2091 \\
\hline $\mathrm{CVr}$ & 1,2769 & 0,5199 & 0,3087 & 0,4732 & 0,5958 \\
\hline
\end{tabular}

$\mathrm{h}^{2} \mathrm{~g}$ : Coeficientes de herdabilidade individual no sentido amplo, livre da interação; $\mathrm{h}^{2} \mathrm{mg}$ : Herdabilidade da média de clone; Acgen: Acurácia genética na seleção de clones; $C^{2}$ bloc: Coeficiente de determinação do bloco; $C^{2}$ int: Coeficiente de determinação da interação genótipos x ambientes; rgloc: Correlação genotípica entre os ambientes; Média geral: Média geral dos caracteres entre os diferentes ambientes; CVgi (\%): Coeficiente de variação genotípica; CVe (\%): Coeficiente de variação experimental; CVr: Coeficiente de variação relativa. *Herdabilidades corrigidas para variância de bloco (herdabilidade ajustada). **Acurácias e herdabilidades de média de clones corrigidas de acordo com o arquivo. Fam

Valor elevado de herdabilidade foi encontrado para a característica profundidade do Pilodyn em Eucalyptus urophylla S.T. Blake, no estudo de Wei e Borralho (1997). Os autores constataram herdabilidade para essa característica igual a $\mathrm{h}^{2} \mathrm{a}=0,64$. Além disso, os autores ressaltam a importância da estimação da densidade básica de eucalipto em um programa de melhoramento, considerando tal característica como a mais importante e representativa da qualidade da madeira e da polpa celulósica.

Os valores de coeficiente de variação genotípico (CVgi \%) encontrados para as características foram expressivos (Tabela 2), sendo possível a seleção de genótipos e a obtenção de ganhos consideráveis (MAIA et al., 2009).

Os valores genéticos para os caracteres de qualidade da madeira foram preditos com alta acurácia (RESENDE; DUARTE, 2007), que variou de 0,8838 a 0,9761, demonstrando que o delineamento experimental e o número de repetições (30) utilizado nos experimentos foram adequados, e que os resultados podem ser considerados fidedignos. A acurácia tem a propriedade de informar sobre o correto ordenamento dos genótipos para fins de seleção e sobre a eficácia da inferência acerca do valor genotípico do clone (RESENDE, 2002).

O coeficiente de variação relativa $(\mathrm{CVr})$ é outra estatística empregada em avaliação genotípica. $\mathrm{O}$ maior $\mathrm{CVr}$ foi de 1,2769 para o caráter Db (Tabela 2), que apresenta alto controle genético, o que pode ter contribuído para um valor de CVr acima da unidade (RESENDE; DUARTE, 2007). O índice DAPxDb apresentou CVr inferior a 1, no entanto, Resende e Duarte (2007) relatam que é possível obter altos valores de acurácia mesmo com CVr's baixos. Segundo esses autores, com 30 a 40 repetições para caracteres de herdabilidade moderada é possível obter acurácias acima de 90\%, mesmo quando a relação $\mathrm{CVg} / \mathrm{CVe}$ é inferior a 0,40. Com isso, valores de CVr devem ser inferidos em conjunto com o número de repetições (RESENDE; DUARTE, 2007).

$\mathrm{Na}$ análise da interação $\mathrm{G}$ x A, para o índice de peso de madeira DAPxDb, a correlação genotípica através dos quatro ambientes ( $\mathrm{rgloc}=0,6743$ ) pode ser considerada moderada, tendendo a alta, de acordo com a classificação de Resende (2007). Dessa forma, é apropriado investigar a adaptabilidade e estabilidade dos clones para esse índice e, se preciso, separar áreas de recomendação de clones, ao invés de considerar os quatro ambientes como um só para esse índice. Constata-se que o índice de peso de madeira criado a partir da multiplicação do DAP com a Db, apresentou maior correla- 
ção genética entre os ambientes se comparado ao volume (rgloc $=0,5030)$. Assim, o caráter DAP é mais estável em relação à característica volume, ou seja, apresenta menor amplitude de variação nas medições. Tal fato pode ser comprovado ao observar na Tabela 2 os maiores valores de CVe (\%) do caráter volume, se comparado aos valores desses parâmetros genéticos para o caráter DAP. Com isso, a medição do DAP é mais precisa do que a do volume, que é uma característica composta por DAP e altura total.

\section{Seleção indireta}

Constata-se a alta correlação genética entre diâmetro à altura do peito (DAP) e volume (Tabela 3). Esse resultado deve-se ao fato do volume ser função quadrática do DAP. Em virtude dessa alta correlação genética, é possível praticar a seleção indireta dos clones de eucalipto visando ganhos em volume, por meio do caráter DAP. Assim, evitam-se erros na medição da altura como: calibragem do aparelho; distância do operador à árvore; balanço da árvore pelo vento, dificultando a visada no topo e; nível de treinamento do operador com o aparelho (COUTO; BASTOS, 1988). A eficiência da seleção indireta foi 1,05, e por esta estimativa constatou-se que $\varphi_{x, y}$ é maior do que a herdabilidade individual do volume. De acordo com Resende (2002), quando isso ocorre, ou seja, quando a eficiência é superior a unidade, a seleção indireta será mais vantajosa que a seleção direta. De acordo com Falconer (1987), a seleção indireta pode promover maiores ganhos em relação à direta se o caráter auxiliar apresentar maior herdabilidade que o principal, e se a correlação genética entre ambos for positiva e de alta magnitude, como é o caso do presente trabalho.

Tabela 3. Correlações genéticas entre os caracteres DAP $(\mathrm{cm})$, altura total $(\mathrm{m})$, volume $\left(\mathrm{m}^{3} \cdot \mathrm{ha}^{-1} \cdot \mathrm{ano}^{-1}\right), \mathrm{Db}(\mathrm{mm})$ e índice DAPXDb para os clones de eucalipto aos três anos de idade, avaliados na análise conjunta entre os ambientes.

Table 3. Genetic correlation between the characters DBH $(\mathrm{cm})$, total height $(\mathrm{m})$, volume $\left(\mathrm{m}^{3} . \mathrm{ha}^{-1} . y e a r^{-1}\right), \mathrm{Bd}(\mathrm{mm})$ and $\mathrm{DBH}$ xBd index for Eucalyptus clones at three years of age evaluated in joint analysis among the environments.

\begin{tabular}{lcccc}
\hline & Atura & Volume & Db & DAPxDb \\
\hline DAP & 0,6629 & 0,9700 & $-0,2783$ & 0,4966 \\
Altura & & 0,7783 & $-0,0053$ & 0,4808 \\
Volume & & $-0,2202$ & 0,5220 \\
Db & & & 0,6786 \\
\hline
\end{tabular}

Considerando esses aspectos, a criação do índice de peso de madeira DAPxDb que une os caracteres de crescimento da árvore e qualidade da madeira, torna-se ideal numa cadeia produtiva de alto rendimento, uma vez que o DAP pode ser medido de modo rápido e confiável, assim como a estimação indireta da densidade básica por meio do Pilodyn (Db). A correlação genética entre DAP e Db foi negativa $(-0,2783)$. Esse fato indica que a seleção unicamente para DAP resultará em declínio para Db e vice-versa. Wei e Borralho (1997) também reportaram um valor negativo $(-0,36)$ de correlação genética entre DAP e Db num estudo com progênies de Eucalyptus urophylla S.T. Blake. Resultados semelhantes ao do presente estudo foram encontrados, também, por Kube et al. (2001), que relataram correlação de -0,57 para DAP e densidade básica medida diretamente em progênies de Eucalyptus nitens (Deane e Maiden). Dessa maneira, os autores citam que os dois caracteres devem ser considerados em combinação num programa de melhoramento para maximização de ganhos genéticos.

\section{Zonas de melhoramento}

Para o índice DAPxDb, a correlação genética entre os ambientes (Tabela 4) Cambará e Fortaleza foi alta $(0,82)$, assim como a correlação genética entre Cambará e São João $(0,70)$, de acordo com o limite estabelecido por Resende (2007). Esse resultado indica que os mesmos clones poderão ser plantados, simultaneamente, em Cambará, Fortaleza e em São João, formando, assim, duas zonas de melhoramento (RESENDE, 2005): Cambará - Fortaleza - São João e Capivara.

Para o ambiente Capivara, um programa de melhoramento distinto deverá ser estabelecido, em virtude da resposta diferenciada do comportamento dos clones nesse local. Essa resposta diferenciada é resultado da interação dos genótipos dos indivíduos com os diversos fatores bióticos e abióticos presentes nesse ambiente, diferindo dos mecanismos de interação ocorridos nos demais 
locais. Portanto, estímulos ambientais controláveis ou não e ocorrência de pragas podem influenciar a performance dos clones em um determinado local. Como elencado na Tabela 1, os ambientes apresentam características similares, exceto para textura do solo, que no ambiente Capivara, exclusivamente, é do tipo arenosa/argilosa. Com isso, a textura do solo aliada a outros fatores podem ter contribuído para o isolamento do local Capivara como uma única zona de melhoramento. Ademais, essa estratificação em zonas de melhoramento distintas é importante para que a interação complexa não comprometa os ganhos genéticos (MAIA et al., 2009; MARTINEZ et al., 2012).

Tabela 4. Correlações genéticas entre os ambientes avaliados par a par, para o índice DAPxDb para os clones de eucalipto aos três anos de idade.

Table 4. Genetic correlation between the environments evaluated in pairs, for DBHxBd index for Eucalyptus clones at three years of age.

\begin{tabular}{lccc}
\hline & Capivara & Fortaleza & São João \\
\hline Cambará & 0,63 & 0,82 & 0,70 \\
Capivara & & 0,56 & 0,65 \\
Fortaleza & & & 0,66 \\
\hline
\end{tabular}

Santos et al. (2015) em um estudo de interação G x A para produtividade em volume ( $\mathrm{m}^{3} \cdot \mathrm{ha}^{-1}$. ano $^{-1}$ ) de clones de eucalipto na mesma rede experimental do presente estudo, reportaram três zonas de melhoramento (Cambará-Fortaleza, Capivara e São João). No presente trabalho, por meio do índice DAPxDb criado, somente duas zonas de melhoramento foram obtidas, representando otimização na seleção de genótipos elite. Assim, enfatiza-se a importância do índice criado, tanto em relação à medição precisa do diâmetro à altura do peito (DAP) quanto da agregação de uma característica de qualidade da madeira, que resultou em redução das zonas de recomendação de clones em relação ao trabalho de Santos et al. (2015). Essa redução ocorreu, pois a medição do DAP é mais precisa do que a do volume, em virtude da não necessidade de estimar a altura da árvore, que está sujeita a diferentes fontes de erros (COUTO; BASTOS, 1988). Além disso, de acordo com os resultados sobre seleção indireta, obtidos no presente estudo, é possível obter ganhos em volume apenas pela avaliação do DAP.

Distintos autores reportam em estudos de interação G x A em eucalipto (RAYMOND et al., 1997; MUNERI; RAYMOND, 2000; OSORIO et al., 2001; HARDNER et al., 2010; SANTOS et al., 2015) que determinar a importância prática e magnitude da interação G x A é crítico para o delineamento de programas de melhoramento e para a tomada de decisões no estabelecimento dos plantios. Além disso, quando há interação G x A significativa é fundamental a separação de áreas específicas para recomendação, plantio ou teste de determinados materiais genéticos. De acordo com Resende (2005), dentro dessas áreas a interação G x A será desprezível e, entre elas a interação não acarretará problemas para o melhorista. Desse modo, cada zona de melhoramento demanda um programa de melhoramento específico, sendo que o número destas zonas indicará o número mínimo de populações de melhoramento a serem empregadas pelos melhoristas (RESENDE, 2005).

Ao analisar em conjunto, o critério de crescimento DAP, que é altamente correlacionado com volume $(0,97)$ e uma característica representativa de qualidade da madeira, a densidade básica estimada indiretamente pela profundidade de penetração do Pilodyn (Db), a seleção de clones de eucalipto pode ser feita de forma eficiente (considerando não só critérios de crescimento, mas também de qualidade da madeira) e tecnicamente correta. Isso ocorre em virtude da não necessidade da medição da altura e eventual estimação do volume, menor número de zonas de melhoramento, rapidez e facilidade na medição do DAP (uma medição direta e precisa) e do caráter Db.

Dessa forma, a seleção de genótipos específicos para cada uma dessas zonas conduzirá à maximização dos ganhos genéticos com seleção em relação ao índice de peso de madeira DAPxDb.

\section{Valores genéticos preditos}

Um ordenamento dos 10 melhores clones para a seleção conjunta entre os quatro ambientes, para o índice de peso de madeira DAPxDb encontra-se na Tabela 5. 
Tabela 5. Ordenamento dos clones de eucalipto aos três anos de idade por seus valores genotípicos e ganhos preditos para o índice DAPxDb, na análise conjunta entre os ambientes.

Table 5. Genotypic values ranking of the Eucalyptus clones at three years of age and predicted gains for DBHxBd index in joint analysis among the environments.

\begin{tabular}{|c|c|c|c|c|c|c|c|c|c|c|c|}
\hline Ordem & Genótipo & g & $u+g$ & Ganho* & $\begin{array}{l}\text { Nova } \\
\text { média }\end{array}$ & $\begin{array}{l}\text { Ganho* } \\
\text { em } \\
\text { relação } \\
\text { à média } \\
\text { geral }\end{array}$ & $\begin{array}{l}\text { Ganho* em } \\
\text { relação à } \\
\text { média da } \\
\text { testemunha }\end{array}$ & Genótipo & $\begin{array}{c}u+ \\
g+ \\
\text { gem }\end{array}$ & $\begin{array}{c}\text { Ganho } \\
\text { individual u } \\
+\mathrm{g}+\text { gem } \\
\text { em relação } \\
\text { à média } \\
\text { geral }\end{array}$ & $\begin{array}{c}\text { Ganho in- } \\
\text { dividual u + } \\
\text { g + gem em } \\
\text { relação à } \\
\text { testemunha }\end{array}$ \\
\hline 1 & 39659 & 1,99 & 8,02 & 1,99 & 8,03 & $32,91 \%$ & $8,73 \%$ & 39095 & 7,87 & $30,31 \%$ & $4,32 \%$ \\
\hline 2 & 32949 & 1,89 & 7,93 & 1,94 & 7,98 & $32,07 \%$ & $8,05 \%$ & 39438 & 7,79 & $28,98 \%$ & $3,25 \%$ \\
\hline 3 & 39410 & 1,86 & 7,90 & 1,91 & 7,95 & $31,66 \%$ & $7,71 \%$ & 34039 & 7,72 & $27,87 \%$ & $2,36 \%$ \\
\hline 4 & 39452 & 1,82 & 7,86 & 1,89 & 7,93 & $31,26 \%$ & $7,38 \%$ & 39352 & 7,69 & $27,32 \%$ & $1,92 \%$ \\
\hline 5 & 39596 & 1,77 & 7,81 & 1,86 & 7,90 & $30,89 \%$ & $7,07 \%$ & 39690 & 7,63 & $26,42 \%$ & $1,20 \%$ \\
\hline 6 & 39465 & 1,77 & 7,81 & 1,85 & 7,89 & $30,63 \%$ & $6,86 \%$ & 39445 & 7,58 & $25,47 \%$ & $0,45 \%$ \\
\hline 7 & 39274 & 1,76 & 7,80 & 1,84 & 7,88 & $30,42 \%$ & $6,69 \%$ & 32864 & 7,54 & $24,92 \%$ & $0,00 \%$ \\
\hline 8 & 38629 & 1,73 & 7,77 & 1,82 & 7,86 & $30,21 \%$ & $6,52 \%$ & 39461 & 7,51 & $24,39 \%$ & $-0,42 \%$ \\
\hline 9 & 6891 & 1,72 & 7,76 & 1,81 & 7,85 & $30,01 \%$ & $6,36 \%$ & 39498 & 7,44 & $23,31 \%$ & $-1,29 \%$ \\
\hline 10 & 39706 & 1,66 & 7,70 & 1,80 & 7,84 & $29,76 \%$ & $6,15 \%$ & 39789 & 7,39 & $22,41 \%$ & $-2,01 \%$ \\
\hline 26 & 32864 & 1,34 & 7,38 & 1,61 & 7,65 & $26,66 \%$ & $3,62 \%$ & - & - & - & - \\
\hline
\end{tabular}

Ganho $(\mathrm{u}+\mathrm{g}+\mathrm{em})$ dos 5 melhores clones em relação à média geral: $28,18 \%$

Ganho $(\mathrm{u}+\mathrm{g}+\mathrm{em})$ dos 5 melhores clones em relação à média da testemunha: $2,61 \%$

Média Geral: 6,04

*Ganho acumulado

Os valores genotípicos (Tabela 5) livres de interação $(\mathrm{u}+\mathrm{g})$ são válidos quando se deseja recomendar tais clones para plantio em qualquer área, desconsiderando o efeito do ambiente. Quando a média dos cinco melhores clones é comparada com a média da população, um ganho relativamente alto de $30,89 \%$ pode ser obtido. No entanto, quando a média desses clones é comparada com a média da testemunha, o ganho é da ordem de 7,07\%. Os ganhos genéticos foram calculados para os efeitos genotípicos médios $(\mathrm{u}+\mathrm{g}+\mathrm{gem}$, em que gem é o efeito médio das interações com locais) (Tabela 5). O ganho em relação à média dos cinco melhores clones se manteve alto se comparado à população de melhoramento sendo da ordem de $28,18 \%$. Em contrapartida, quando comparou-se a média desses mesmos clones com a média da testemunha, o ganho foi de 2,61\%. Tal fato evidencia a plasticidade do genótipo 32864, que foi classificado na $7^{\mathrm{a}}$ posição (Tabela 5), e a necessidade de recorrer a novas fontes de germoplasma.

\section{Estabilidade e adaptabilidade}

Os resultados sobre a estabilidade (MHVG), adaptabilidade (PRVG) e estabilidade, adaptabilidade e produtividade simultaneamente (MHPRVG*MG) referentes à característica peso de madeira (DAPxDb) são apresentados na Tabela 6.

Os valores de PRVG e MHPRVG indicam a superioridade média do genótipo em relação à média do ambiente em que for plantado (RESENDE, 2007). Considerando esses aspectos, o clone 39095 responde 1,298 vezes a média do ambiente em que for plantado (Tabela 6). Os cinco melhores clones, em relação aos critérios PRVG, MHVG e MHPRVG coincidem totalmente com o ordenamento de efeitos genotípicos médios entre os ambientes (Tabela 5). A coincidência foi de $100 \%$ dentre os cinco melhores clones e houve inversão de ordem dentre os coincidentes. Esse resultado mostra a equivalência do método utilizado no presente estudo, já que assim como o ordenamento por $\mathrm{u}+\mathrm{g}$ + gem foi similar ao do MHPRVG*MG, os ganhos foram semelhantes.

O ganho com a seleção dos cinco melhores clones $(39095,39438,34039,39352,39690) \mathrm{em}$ relação à população de melhoramento para o critério $M H P R V G{ }^{*} M G$ foi de 27,69\% e em relação à testemunha foi 2,19\%. Apesar de ganho alto em relação à população, o ganho em relação à testemunha (32864) foi, mais uma vez, baixo. Para u + g + gem, ganhos de 28,18\% e 2,61\% (Tabela 5) foram obtidos. Assim, apesar de semelhantes os ganhos por $\mathrm{u}+\mathrm{g}+$ gem e MHPRVG* MG, este último critério possibilitou ganhos ligeiramente menores. Tal fato pode ser explicado, uma vez que o método MHPRVG* MG, além de apresentar os valores genotípicos acrescidos por um efeito médio 
Nunes et al. - Estabelecimento de zonas de melhoramento para clones de eucalipto no Rio Grande do Sul

entre os quatro ambientes, ele também fornece os valores genéticos já descontados (penalizados) da instabilidade e capitalizados pela adaptabilidade (RESENDE, 2007). Dessa forma, ressalta-se a importância do estudo da estabilidade e adaptabilidade em caracteres com tendência de moderada a alta correlação genética entre ambientes, como é o caso do índice de peso de madeira DAPxDb $($ rgloc $=0,6743$, Tabela 2$)$.

Tabela 6. Ordenamento da estabilidade de valores genéticos (MHVG), adaptabilidade de valores genéticos (PRVG) e estabilidade e adaptabilidade simultaneamente (MHPRVG) para o índice DAPxDb dos clones de eucalipto aos três anos de idade.

Table 6. Ranking of stability of genetic values (MHVG), adaptability of genetic values (PRVG) and stability and adaptability simultaneously (MHPRVG) for DBHxBd index of the Eucalyptus clones at three years of age.

\begin{tabular}{ccc|ccc|ccc}
\hline \multicolumn{3}{c|}{ Estabilidade } & \multicolumn{3}{c|}{ Adaptabilidade } & \multicolumn{3}{c}{ Estabilidade e Adaptabilidade } \\
\hline Ordem & Genótipo & MHVG & Genótipo & PRVG & PRVG*MG & Genótipo & MHPRVG & MHPRVG*MG \\
\hline 1 & $\underline{39095}$ & 7,758 & $\underline{39095}$ & 1,300 & 7,854 & $\underline{39095}$ & 1,298 & 7,842 \\
2 & $\underline{34039}$ & 7,689 & $\underline{39438}$ & 1,285 & 7,764 & $\underline{39438}$ & 1,283 & 7,751 \\
3 & $\underline{39438}$ & 7,659 & $\underline{34039}$ & 1,283 & 7,751 & $\underline{34039}$ & 1,279 & 7,726 \\
4 & $\underline{39352}$ & 7,575 & $\underline{39352}$ & 1,270 & 7,671 & $\underline{39352}$ & 1,268 & 7,658 \\
5 & $\underline{39690}$ & 7,515 & $\underline{39690}$ & 1,263 & 7,631 & $\underline{39690}$ & 1,256 & 7,586 \\
6 & 32864 & 7,512 & 32864 & 1,253 & 7,573 & 32864 & 1,249 & 7,548 \\
7 & 39461 & 7,431 & 39445 & 1,250 & 7,552 & 39445 & 1,244 & 7,514 \\
8 & 39445 & 7,425 & 39461 & 1,243 & 7,512 & 39461 & 1,241 & 7,496 \\
9 & 39498 & 7,335 & 39498 & 1,229 & 7,427 & 39498 & 1,228 & 7,419 \\
10 & 38903 & 7,320 & 38903 & 1,221 & 7,379 & 39424 & 1,219 & 7,364 \\
\hline
\end{tabular}

Ganho com relação à média geral (5 melhores): $27,69 \%$

Ganho com relação à média da testemunha (5 melhores): $2,19 \%$

Obs: Clones sublinhados são os cinco melhores do ordenamento de efeitos genotípicos médios ( $u+g+$ gem) na seleção conjunta entre os ambientes, presentes também no ordenamento da seleção para estabilidade, adaptabilidade e estabilidade e adaptabilidade (15 em I5, 100\%).

\section{CONCLUSÕES}

A alta correlação genética entre DAP e volume possibilita a seleção indireta do volume por meio do DAP, com eficiência seletiva maior que a unidade.

Com o uso do índice de peso de madeira DAPxDb é possível efetuar a seleção de genótipos superiores de modo eficiente e com apenas duas zonas de melhoramento, considerando os quatro locais estudados.

Apesar dos cinco clones selecionados para os critérios de adaptabilidade e estabilidade, a testemunha comercial apresenta considerável plasticidade genética em relação à população de melhoramento.

\section{AGRADECIMENTOS}

À Universidade Federal de Viçosa, à Empresa CMPC Celulose Riograndense, ao CNPq e à Capes.

\section{REFERENCIAS BIBLIOGRÁFICAS}

CAIXETA, R. P.; TRUGILHO, P. F.; ROSADO, S. C. S.; LIMA, J. T. Propriedades e classificação da madeira aplicada à seleção de genótipos de Eucalyptus. Revista Árvore, Viçosa, v. 27, n. 1, p. 43-51, 2003.

COUTO, H. T. Z.; BASTOS, N. L. M. Erros de medição de altura em povoamentos de Eucalyptus em região plana. IPEF, Piracicaba, n. 39, p. 21-31, 1988.

CRUZ, C. D.; REGAZZI, A. J. Modelos biométricos aplicados ao melhoramento genético. 2.ed. Viçosa: UFV, 1994. $390 \mathrm{p}$.

CRUZ, C. D.; REGAZZI, A. J.; CARNEIRO, P. C. S. Modelos biométricos aplicados ao melhoramento genético, Viçosa, Editora UFV, 2004, v. 1, p. 171-201. 
FALCONER, D. S. Introdução à genética quantitativa. Tradução de M. A. Silva e J. C. Silva. Viçosa: UFV, Imprensa Universitária, 1987. 279 p.

GOLDENBERG, J. B. El empleo de la correlación en el mejoramento genético de las plantas. Fitotecnia Latinoamericana, v. 5, n. 2, p. 1-8, 1968.

GOMIDE, J. L.; FANTUZZI NETO, H.; REGAZZI, A. J. Análise de critérios de qualidade da madeira de eucalipto para produção de celulose kraft. Revista Árvore, Viçosa, v. 34, n. 2, p. 339-344, 2010.

GREAVES, B. L.; BORRALHO, N. M. G.; RAYMOND, C. A.; FARRINGTON, A. Use of a Pilodyn for the indirect selection of basic density in Eucalyptus nitens. Canadian Journal of Forest Research, Ottawa, v. 26, n. 9, p. 1643-1650, 1996.

HARDNER, C. M.; DIETERS, M.; DALE, G.; DELACY, I.; BASFORD, K. E. Patterns of genotype-by-environment interaction in diameter at breast height at age 3 for eucalypt hybrid clones grown for reafforestation of lands affected by salinity. Tree Genetics \& Genomes, v. 6, n. 6, p. 833-851, 2010.

HARDNER, C.; DIETERS, M.; DELACY, I.; NEAL, J.; FLETCHER, S.; DALE, G.; BASFORD, K. Identifying deployment zones for Eucalyptus camaldulensis $\mathrm{x}$ E. globulus and $\mathrm{x}$ E. grandis hybrids using factor analytic modelling of genotype by environment interaction. Australian Forestry, Melbourne, v. 74, n. 1, p. 30-35, 2011.

KUBE, P. D.; RAYMOND, C. A.; BANHAM, P. W. Genetic parameters for diameter, basic density, cellulose content and fibre properties for Eucalyptus nitens. Forest Genetics, Zvolen, v. 8, n. 4, p. 285-294, 2001.

LEITE, H. G.; GUIMARÃES, D. P.; CAMPOS, J. C. C. Descrição e emprego de um modelo para estimar múltiplos volumes de árvores. Revista Árvore, Viçosa, v. 19, n. 1, p. 75-79, 1995.

MAIA, M. C. C.; RESENDE, M. D. V.; PAIVA, J. R.; CAVALCANTI, J. J. V.; BARROS, L. M. Seleção simultânea para produção, adaptabilidade e estabilidade genotípicas em clones de cajueiro, via modelos mistos. Pesquisa Agropecuária Tropical, Goiânia, v. 39, n. 1, p. 43-50, 2009.

MARTINEZ, D. T.; RESENDE, M. D. V.; COSTA, R. B.; HIGA, A. R.; SANTOS, G. A.; FIER, I. S. N. Estudo da interação genótipo $\mathrm{x}$ ambiente em progênies de Pinus taeda por meio da análise de parâmetros genéticos. Floresta, Curitiba, v. 42, n. 3, p. 539-552, 2012.

MITCHELL, H. L. A concept of intrinsic wood quality, and nondestructive methods for determining quality in standing timber. Wisconsin: USDA Forest Service, 1961. 25 p. (FPL, 2233)

MUNERI, A.; RAYMOND, C. A.; Genetic parameters and genotype-by-environment interactions for basic density, pilodyn penetration and stem diameter in Eucalyptus globulus. Forest Genetics, Zvolen, v. 7, n. 4, p. 317-328, 2000.

OSORIO, L. F.; WHITE, T. L.; HUBER, D. A. Age Trends of Heritabilities and Genotype-by-Environment Interactions for Growth Traits and Wood Density from Clonal Trials of Eucalyptus grandis Hill ex Maiden. Silvae Genetica, Frankfurt, v. 50, n.1, p. 30-37, 2001.

PROTÁSIO, T. P.; GOULART, S. L.; NEVES, T. A.; TRUGILHO, P. F.; RAMALHO, F. M. G.; QUEIROZ, L. M. R. S. B. Qualidade da madeira e do carvão vegetal oriundos de floresta plantada em Minas Gerais. Pesquisa Florestal Brasileira, Colombo, v. 34, n. 78, p. 111-123, 2014.

RAYMOND, C. A.; VOLKER, W.; WILLIAMS, E. R. Provenance variation, genotype by environment interactions and age-age correlations for Eucalyptus regnans on nine sites in south eastern Australia. Forest Genetics, Zvolen, v. 4, n. 4, p. 235-251, 1997. 
Nunes et al. - Estabelecimento de zonas de melhoramento para clones de eucalipto no Rio Grande do Sul

RESENDE, M. D. V. Genética Biométrica e Estatística no Melhoramento de Plantas Perenes. Brasília: Embrapa informações tecnológicas, 2002. 975 p.

RESENDE, M. D. V. Matemática e estatística na análise de experimentos e no melhoramento genético. Colombo: Embrapa Florestas, 2007. 562 p.

RESENDE, M. D. V. Melhoramento de Essências Florestais. In: BOREM, A. Melhoramento de Espécies Cultivadas. Viçosa: Editora UFV, 2005. p. 717-780.

RESENDE, M. D. V. Novas abordagens estatísticas na análise de experimentos e campo. Colombo: Embrapa Florestas, 2004. 60 p. (Documentos, 100).

RESENDE, M. D. V.; DUARTE, J. B. Precisão e controle de qualidade em experimentos de avaliação de cultivares. Pesquisa Agropecuária Tropical, Goiânia, v. 37, n. 3, p. 182-194, 2007.

SANTOS, G. A.; RESENDE, M. D. V.; SILVA, L. D.; HIGA, A.; ASSIS, T. F. Adaptabilidade de híbridos multiespécies de Eucalyptus ao Estado do Rio Grande do Sul. Revista Árvore, Viçosa, v. 37, n. 4, p. 759-769, 2013.

SANTOS, G. A.; RESENDE, M. D. V.; SILVA, L. D.; HIGA, A.; ASSIS, T. F. Interação genótipos x ambientes para produtividade de clones de Eucalyptus no Estado do Rio Grande do Sul. Revista Árvore, Viçosa, v. 39, n. 1, p. 81-91, 2015.

VENCOVSKY, R.; BARRIGA, P. Genética biométrica no fitomelhoramento. Ribeirão Preto: Sociedade Brasileira de Genética, p. 486, 1992.

WEI, X.; BORRAlHO, N. M. G. Genetic Control of Wood Basic Density and Bark Thickness and Their Relationships with Growth Traits of Eucalyptus urophylla in South East China. Silvae Genetica, Frankfurt, v. 46, n. 4, p. 245-50, 1997.

Recebido em 11/05/2015

Aceito para publicação em 13/01/2016 